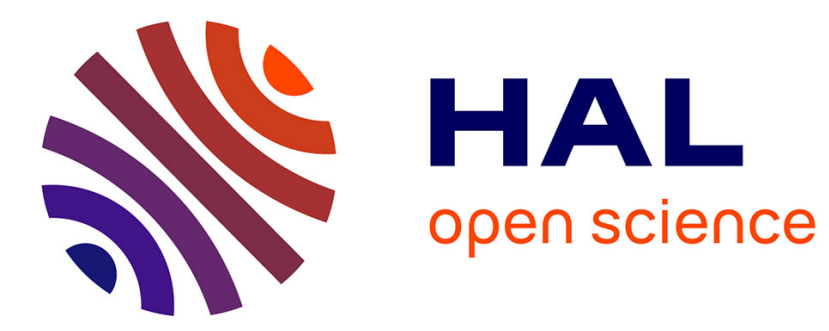

\title{
Particle approximation of a constrained model for traffic flow
}

\author{
Florent Berthelin, Paola Goatin
}

\section{To cite this version:}

Florent Berthelin, Paola Goatin. Particle approximation of a constrained model for traffic flow. Nonlinear Differential Equations and Applications, 2017, 24 (5), pp.24-55. hal-01437867

\section{HAL Id: hal-01437867 https://hal.science/hal-01437867}

Submitted on 17 Jan 2017

HAL is a multi-disciplinary open access archive for the deposit and dissemination of scientific research documents, whether they are published or not. The documents may come from teaching and research institutions in France or abroad, or from public or private research centers.
L'archive ouverte pluridisciplinaire HAL, est destinée au dépôt et à la diffusion de documents scientifiques de niveau recherche, publiés ou non, émanant des établissements d'enseignement et de recherche français ou étrangers, des laboratoires publics ou privés. 


\title{
Particle approximation of a constrained model for traffic flow
}

\author{
Florent Berthelin and Paola Goatin
}

To Prof. Alberto Bressan

\begin{abstract}
We rigorously prove the convergence of the micro-macro limit for particle approximations of the Aw-Rascle-Zhang equations with a maximal density constraint. The lack of BV bounds on the density variable is supplied by a compensated compactness argument.
\end{abstract}

Mathematics Subject Classification (2010). Primary 35L87; Secondary 35L75, 35A35.

Keywords. Conservation laws with density constraint - Many-particle system - Traffic flow models.

\section{Introduction}

Macroscopic traffic flow models usually consist of partial differential equations describing the evolution of aggregated quantities, like traffic density and mean velocity. They express the mass conservation and eventually the traffic acceleration. In this article, we focus on a pressure-less gas dynamics system subject to a maximal density constraint, which was introduced in [5] and can be derived through a singular limit in the pressure term of a modified Aw-Rascle-Zhang model [2, 12].

In the following, we denote by $\rho, v$ the density and velocity of the traffic and by $p$ the "reserve" of velocity acting as an anticipation factor of drivers to the local traffic conditions. We consider the following system of conservation laws

$$
\left\{\begin{array}{l}
\partial_{t} \rho+\partial_{x}(\rho v)=0 \\
\partial_{t}(\rho(v+p))+\partial_{x}(\rho v(v+p))=0, \quad t>0, x \in \mathbb{R}
\end{array}\right.
$$

subject to the constraints

$$
0 \leq \rho(t, x) \leq \rho^{*}, \quad p(t, x) \geq 0, \quad\left(\rho(t, x)-\rho^{*}\right) p(t, x)=0 \quad \text { a.e. } t, x,
$$


for some $\rho^{*} \in \mathbb{R}^{+}$denoting the maximal density of cars allowed on the road. System (1.1) is equipped with the following initial data

$$
\rho(0, x)=\rho^{0}(x), \quad v(0, x)=v^{0}(x), \quad p(0, x)=p^{0}(x), \quad x \in \mathbb{R} .
$$

We assume that

(H1) $\rho^{0} \in L^{1}(\mathbb{R}) \cap L^{\infty}(\mathbb{R})$ with $0 \leq \rho^{0} \leq \rho^{*}$ and $\rho^{0}$ with compact support;

(H2) $v^{0}, p^{0} \in L^{\infty}(\mathbb{R}) \cap \mathrm{BV}(\mathbb{R})$ with $v^{0} \geq 0, p^{0} \geq 0$ and $\left(\rho^{0}(x)-\rho^{*}\right) p^{0}(x)=0$ for a.e. $x \in \mathbb{R}$.

In [5], the authors introduced the following constrained follow-the-leader model to compute approximate solutions of (1.1)-(1.3).

Let us denote by $x_{i}(t), V_{i}(t)$ and $p_{i}(t)$ the position, speed and reserve of velocity, respectively, of the $i$-th particle at time $t \geq 0$, for $0 \leq i \leq N$. The initial conditions

$$
x_{i}^{N}(0)=\bar{x}_{i}^{N}, \quad V_{i}^{N}(0)=\bar{V}_{i}^{N}, \quad p_{i}^{N}(0)=\bar{p}_{i}^{N} \quad \text { for } i=0, \ldots, N,
$$

are defined as follows: let $x_{\min }<x_{\max }$ the extremal points of the convex hull of the support of $\rho^{0}$, so that

$$
\operatorname{Supp}\left(\rho^{0}\right) \subseteq\left[x_{\min }, x_{\max }\right],
$$

and set

$$
\begin{gathered}
l_{N}=\frac{1}{N} \int_{\mathbb{R}} \rho^{0}(x) d x, \quad d_{N}=l_{N} / \rho^{*}, \\
\bar{x}_{0}^{N}=x_{\min }, \quad \bar{x}_{i}^{N}=\sup \left\{x \in \mathbb{R} ; \int_{\bar{x}_{i-1}^{N}}^{x} \rho^{0}(x) d x<l_{N}\right\}, \text { for } i=0, \ldots, N, \\
\bar{V}_{i}^{N}=\sup _{\left[\bar{x}_{i}^{N}, \bar{x}_{i+1}^{N}[\right.} v^{0}, \quad \bar{p}_{i}^{N}=\sup _{\left[\bar{x}_{i}^{N}, \bar{x}_{i+1}^{N}[\right.} p^{0}, \quad \text { for } i=0, \ldots, N-1, \\
\bar{V}_{N}^{N}=\sup _{\left[\bar{x}_{N}^{N},+\infty[\right.} v^{0}, \quad \bar{p}_{i}^{N}=\sup _{\left[\bar{x}_{N}^{N},+\infty[\right.} p^{0} .
\end{gathered}
$$

Notice that from (1.7) we get $\bar{x}_{N}^{N}=x_{\max }$ and

$$
\int_{\mathbb{R}} \rho^{0}(x) d x=\int_{x_{\min }}^{x_{\max }} \rho^{0}(x) d x=\sum_{i=1}^{N} \int_{\bar{x}_{i-1}^{N}}^{\bar{x}_{i}^{N}} \rho^{0}(x) d x=N l_{N},
$$

since

$$
l_{N}=\int_{\bar{x}_{i-1}^{N}}^{\bar{x}_{i}^{N}} \rho^{0}(x) d x, \quad i=1, \ldots, N .
$$

Notice also that we have

$$
l_{N}=\int_{\bar{x}_{i-1}^{N}}^{\bar{x}_{i}^{N}} \rho^{0}(x) d x \leq\left\|\rho^{0}\right\|_{\infty}\left(\bar{x}_{i}^{N}-\bar{x}_{i-1}^{N}\right) \leq \rho^{*}\left(\bar{x}_{i}^{N}-\bar{x}_{i-1}^{N}\right)
$$

for all $i=1, \ldots, N$, and therefore

$$
\bar{x}_{i}^{N}-\bar{x}_{i-1}^{N} \geq d_{N}, \quad i=1, \ldots, N .
$$

The dynamics of the discrete model is the following: each particle moves freely until it reaches the minimal distance to the preceding one, that is to 
say $x_{i+1}^{N}(t)-x_{i}^{N}(t)=d_{N}$. At this point, the particle $i$ takes the velocity of the particle $i+1$ and they keep the distance $d_{N}$ forever. For any initial positions and velocities of the $N+1$ particles, these "interactions" can only happen $k$ times, with $k \leq N$. Let us denote by $t_{1} \leq t_{2} \leq \ldots<t_{k}$ the times when an interaction happens and we denotes by $i_{m}$ the number of particle(s) for which at time $t_{m}$, the collision is between the $i_{m}$-th and the $\left(i_{m}+1\right)$-th particles. The particle dynamics is therefore described by the following rules

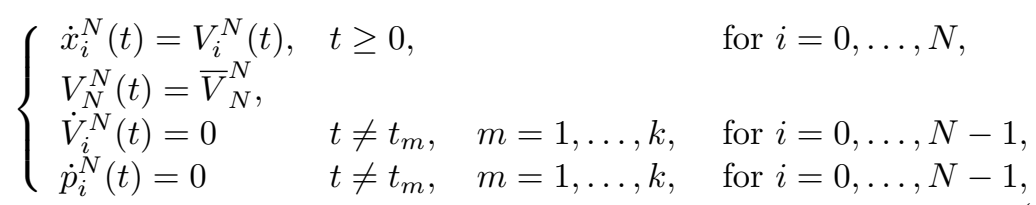

and at times $t_{m}$, there is a jump such that for $t \geq t_{m}$,

$$
\left\{\begin{array}{l}
V_{i_{m}}^{N}(t):=V_{i_{m}+1}^{N}(t), \\
p_{i(m)}^{N}(t):=V_{i_{m}}^{N}\left(t_{m}-\right)-V_{i_{m}}^{N}\left(t_{m}+\right)+p_{i_{m}}^{N}\left(t_{m}-\right) .
\end{array} \quad t \geq t_{m},\right.
$$

We introduce the variables

$$
y_{i}^{N}(t)=\frac{l_{N}}{x_{i+1}^{N}(t)-x_{i}^{N}(t)}, \quad i=0, \ldots, N-1,
$$

which satisfy

$$
\dot{y}_{i}^{N}(t)=-\frac{l_{N}\left(\dot{x}_{i+1}^{N}(t)-\dot{x}_{i}^{N}(t)\right)}{\left(x_{i+1}^{N}(t)-x_{i}^{N}(t)\right)^{2}}=-\frac{y_{i}^{N}(t)^{2}}{l_{N}}\left(V_{i+1}^{N}(t)-V_{i}^{N}(t)\right) .
$$

Since $x_{i}^{N}(t)-x_{i-1}^{N}(t) \geq d_{N}$, we have $y_{i}^{N}(t) \leq l_{N} / d_{N}=\rho^{*}$.

We define the piecewise constant density $\hat{\rho}^{N}$ by

$$
\hat{\rho}^{N}(t, x)=\sum_{i=0}^{N-1} y_{i}^{N}(t) \mathbf{I}_{\left[x_{i}^{N}(t), x_{i+1}^{N}(t)[\right.}(x),
$$

the velocity $\hat{v}^{N}$ by

$$
\hat{\rho}^{N} \hat{v}^{N}(t, x)=\sum_{i=0}^{N-1} y_{i}^{N}(t) V_{i}^{N}(t) \mathbf{I}_{\left[x_{i}^{N}(t), x_{i+1}^{N}(t)[\right.}(x),
$$

and the pressure term $\hat{p}^{N}$ by

$$
\hat{\rho}^{N} \hat{p}^{N}(t, x)=\sum_{i=0}^{N-1} y_{i}^{N}(t) p_{i}^{N}(t) \mathbf{I}_{\left[x_{i}^{N}(t), x_{i+1}^{N}(t)[\right.}(x) .
$$

Remark 1.1. These definitions identify $\hat{v}^{N}$ and $\hat{p}^{N}$ where $\hat{\rho}^{N} \neq 0$, that is to say away from vacuum. Thus we need to extend the functions $\hat{v}^{N}$ and $\hat{p}^{N}$ when $\hat{\rho}^{N}=0$. While the pressure term must be equal to zero by $(1.2)$, the velocity is given any non-negative constant value that does not increase the total variation. For example, by taking the average between two no-vacuum zones and extending by constants at infinity. 
The main result of the present article is the convergence of the microscopic constrained follow-the-leader model to the macroscopic constrained Aw-Rascle-Zhang system as the number of particles tends to infinity.

Theorem 1.2. Let $\rho^{0}, v^{0}$ and $p^{0}$ satisfy (H1)-(H2) and consider the discrete quantities $\left(\hat{\rho}^{N}, \hat{v}^{N}, \hat{p}^{N}\right)$ defined by (1.14)-(1.16) with (1.12) and (1.4)-(1.8). Then there exists $(\rho, v, p)$ with $\rho \in L^{1}(\mathbb{R}) \cap L^{\infty}(\mathbb{R})$ and $v, p \in L^{\infty}(\mathbb{R}) \cap B V(\mathbb{R})$, solution of (1.1) with the constraints (1.2), with initial data $\left(\rho^{0}, v^{0}, p^{0}\right)$ such that, up to a subsequence,

$$
\hat{\rho}^{N} \rightarrow \rho, \quad \hat{\rho}^{N} \hat{v}^{N} \rightarrow \rho v, \quad \hat{\rho}^{N} \hat{p}^{N} \rightarrow \rho p
$$

in the distributional sense.

The proof is deferred to Section 4.3. We recall that previous derivations of macroscopic traffic models from microscopic dynamical systems have been investigated for the classical Lighthill-Whitham-Richards equation $[7,8]$ and its non-local version [11], for the Aw-Rascle system [1, 9], for a phasetransition model based on a speed bound [6], and for Hughes model of crowd motion [10]. In our case, the main difficulty is represented by the lack of a uniform bound on the density total variation, that cannot be compensated by the compactness of the Riemann invariants like in [9], due to the zeropressure term in the momentum equation. Therefore, the convergence relies on a compensated compactness argument introduced in [3].

The paper is organized as follows. In Section 2 we provide the convergence proof for initial data. Section 3 collects the $L^{\infty}$ and $B V$ estimates satisfied by the approximate solutions, which allow to show their convergence in Section 4.

\section{Initial data limit}

We start first by proving that the discrete quantities constructed at the previous section are compatible with the initial data.

Proposition 2.1. Let $\rho^{0}, v^{0}$ and $p^{0}$ satisfy (H1)-(H2). We consider the discrete quantities (1.14)-(1.16) with (1.12) and (1.4)-(1.8). Then, for all $\varphi \in \mathcal{C}_{c}^{\infty}(\mathbb{R})$, we have

$$
\begin{gathered}
\int_{\mathbb{R}} \hat{\rho}^{N}(0, x) \varphi(x) d x \underset{N \rightarrow+\infty}{\rightarrow} \int_{\mathbb{R}} \rho^{0}(x) \varphi(x) d x \\
\int_{\mathbb{R}} \hat{\rho}^{N}(0, x) \hat{v}^{N}(0, x) \varphi(x) d x \underset{N \rightarrow+\infty}{\rightarrow} \int_{\mathbb{R}} \rho^{0}(x) v^{0}(x) \varphi(x) d x
\end{gathered}
$$

and

$$
\int_{\mathbb{R}} \hat{\rho}^{N}(0, x) \hat{p}^{N}(0, x) \varphi(x) d x \underset{N \rightarrow+\infty}{\rightarrow} \int_{\mathbb{R}} \rho^{0}(x) p^{0}(x) \varphi(x) d x .
$$

Proof. We have

$$
\int_{\mathbb{R}} \hat{\rho}^{N}(0, x) \varphi(x) d x=\sum_{i=0}^{N-1} \int_{\bar{x}_{i}^{N}}^{\bar{x}_{i+1}^{N}} \frac{l_{N}}{\bar{x}_{i+1}^{N}-\bar{x}_{i}^{N}} \varphi(x) d x
$$




$$
=\sum_{i=0}^{N-1} l_{N} \varphi\left(\bar{x}_{i}^{N}\right)+\sum_{i=0}^{N-1} \frac{l_{N}}{\bar{x}_{i+1}^{N}-\bar{x}_{i}^{N}} \int_{\bar{x}_{i}^{N}}^{\bar{x}_{i+1}^{N}}\left(\varphi(x)-\varphi\left(\bar{x}_{i}^{N}\right)\right) d x .
$$

Using (1.9), we get

$$
\begin{aligned}
\sum_{i=0}^{N-1} l_{N} \varphi\left(\bar{x}_{i}^{N}\right) & =\sum_{i=0}^{N-1} \int_{\bar{x}_{i}^{N}}^{\bar{x}_{i+1}^{N}} \rho^{0}(x) \varphi\left(\bar{x}_{i}^{N}\right) d x \\
& =\sum_{i=0}^{N-1} \int_{\bar{x}_{i}^{N}}^{\bar{x}_{i+1}^{N}} \rho^{0}(x)\left(\varphi(x)+\varphi\left(\bar{x}_{i}^{N}\right)-\varphi(x)\right) d x \\
& =\int_{\mathbb{R}} \rho^{0}(x) \varphi(x) d x+\sum_{i=0}^{N-1} \int_{\bar{x}_{i}^{N}}^{\bar{x}_{i+1}^{N}} \rho^{0}(x)\left(\varphi\left(\bar{x}_{i}^{N}\right)-\varphi(x)\right) d x
\end{aligned}
$$

Therefore

$$
\begin{aligned}
\int_{\mathbb{R}} \hat{\rho}^{N}(0, x) \varphi(x) d x & -\int_{\mathbb{R}} \rho^{0}(x) \varphi(x) d x \\
= & \sum_{i=0}^{N-1} \int_{\bar{x}_{i}^{N}}^{\bar{x}_{i+1}^{N}} \rho^{0}(x)\left(\varphi\left(\bar{x}_{i}^{N}\right)-\varphi(x)\right) d x \\
& +\sum_{i=0}^{N-1} \frac{l_{N}}{\bar{x}_{i+1}^{N}-\bar{x}_{i}^{N}} \int_{\bar{x}_{i}^{N}}^{\bar{x}_{i+1}^{N}}\left(\varphi(x)-\varphi\left(\bar{x}_{i}^{N}\right)\right) d x .
\end{aligned}
$$

Now

$$
\begin{aligned}
& \left|\int_{\mathbb{R}} \hat{\rho}^{N}(0, x) \varphi(x) d x-\int_{\mathbb{R}} \rho^{0}(x) \varphi(x) d x\right| \\
\leq & \left\|\varphi^{\prime}\right\|_{\infty} \sum_{i=0}^{N-1} \int_{\bar{x}_{i}^{N}}^{\bar{x}_{i+1}^{N}} \rho^{0}(x)\left|\bar{x}_{i}^{N}-x\right| d x+\left\|\varphi^{\prime}\right\|_{\infty} \sum_{i=0}^{N-1} \frac{l_{N}}{\bar{x}_{i+1}^{N}-\bar{x}_{i}^{N}} \int_{\bar{x}_{i}^{N}}^{\bar{x}_{i+1}^{N}}\left(x-\bar{x}_{i}^{N}\right) d x \\
\leq & \left\|\varphi^{\prime}\right\|_{\infty} \sum_{i=0}^{N-1}\left(\bar{x}_{i+1}^{N}-\bar{x}_{i}^{N}\right) \int_{\bar{x}_{i}^{N}}^{\bar{x}_{i+1}^{N}} \rho^{0}(x) d x+\left\|\varphi^{\prime}\right\|_{\infty} l_{N} \sum_{i=0}^{N-1} \frac{\bar{x}_{i+1}^{N}-\bar{x}_{i}^{N}}{2} \\
\leq & \left\|\varphi^{\prime}\right\|_{\infty} l_{N} \frac{3}{2} \sum_{i=0}^{N-1}\left(\bar{x}_{i+1}^{N}-\bar{x}_{i}^{N}\right) \\
\leq & l_{N} \frac{3}{2}\left\|\varphi^{\prime}\right\|_{\infty}\left(\bar{x}_{N}^{N}-\bar{x}_{0}^{N}\right) \underset{N \rightarrow+\infty}{\rightarrow} 0 . \\
\leq & l_{N} \frac{3}{2}\left\|\varphi^{\prime}\right\|_{\infty}\left(x_{\max }-x_{\min }\right) \underset{N \rightarrow+1}{\rightarrow}
\end{aligned}
$$

Thus we get

$$
\int_{\mathbb{R}} \hat{\rho}^{N}(0, x) \varphi(x) d x \underset{N \rightarrow+\infty}{\rightarrow} \int_{\mathbb{R}} \rho^{0}(x) \varphi(x) d x .
$$

We consider now now the product $\hat{\rho}^{N}(0, x) \hat{v}^{N}(0, x)$. In this case we have the relation

$$
\int_{\mathbb{R}} \hat{\rho}^{N}(0, x) \hat{v}^{N}(0, x) \varphi(x) d x
$$




$$
\begin{aligned}
& =\sum_{i=0}^{N-1} \bar{V}_{i}^{N} \frac{l_{N}}{\bar{x}_{i+1}^{N}-\bar{x}_{i}^{N}} \int_{\bar{x}_{i}^{N}}^{\bar{x}_{i+1}^{N}} \varphi(x) d x \\
& =\sum_{i=0}^{N-1} \bar{V}_{i}^{N} l_{N} \varphi\left(\bar{x}_{i}^{N}\right)+\sum_{i=0}^{N-1} \bar{V}_{i}^{N} \frac{l_{N}}{\bar{x}_{i+1}^{N}-\bar{x}_{i}^{N}} \int_{\bar{x}_{i}^{N}}^{\bar{x}_{i+1}^{N}}\left(\varphi(x)-\varphi\left(\bar{x}_{i}^{N}\right)\right) d x
\end{aligned}
$$

and

$$
\begin{aligned}
\sum_{i=0}^{N-1} \bar{V}_{i}^{N} l_{N} \varphi\left(\bar{x}_{i}^{N}\right)= & \sum_{i=0}^{N-1} \bar{V}_{i}^{N} \int_{\bar{x}_{i}^{N}}^{\bar{x}_{i+1}^{N}} \rho^{0}(x) \varphi\left(\bar{x}_{i}^{N}\right) d x \\
= & \sum_{i=0}^{N-1} \int_{\bar{x}_{i}^{N}}^{\bar{x}_{i+1}^{N}} \bar{V}_{i}^{N} \rho^{0}(x)\left(\varphi(x)+\varphi\left(\bar{x}_{i}^{N}\right)-\varphi(x)\right) d x \\
= & \int_{\mathbb{R}} \rho^{0}(x) v^{0}(x) \varphi(x) d x+\sum_{i=0}^{N-1} \int_{\bar{x}_{i}^{N}}^{\bar{x}_{i+1}^{N}}\left(\bar{V}_{i}^{N}-v^{0}(x)\right) \rho^{0}(x) \varphi(x) d x \\
& +\sum_{i=0}^{N-1} \int_{\bar{x}_{i}^{N}}^{\bar{x}_{i+1}^{N}} \bar{V}_{i}^{N} \rho^{0}(x)\left(\varphi\left(\bar{x}_{i}^{N}\right)-\varphi(x)\right) d x .
\end{aligned}
$$

Therefore

$$
\begin{aligned}
& \int_{\mathbb{R}} \hat{\rho}^{N}(0, x) \hat{v}^{N}(0, x) \varphi(x) d x-\int_{\mathbb{R}} \rho^{0}(x) v^{0}(x) \varphi(x) d x \\
& =\sum_{i=0}^{N-1} \bar{V}_{i}^{N} \int_{\bar{x}_{i}^{N}}^{\bar{x}_{i+1}^{N}} \rho^{0}(x)\left(\varphi\left(\bar{x}_{i}^{N}\right)-\varphi(x)\right) d x \\
& \quad+\sum_{i=0}^{N-1} \bar{V}_{i}^{N} \frac{l_{N}}{\bar{x}_{i+1}^{N}-\bar{x}_{i}^{N}} \int_{\bar{x}_{i}^{N}}^{\bar{x}_{i+1}^{N}}\left(\varphi(x)-\varphi\left(\bar{x}_{i}^{N}\right)\right) d x \\
& \quad+\sum_{i=0}^{N-1} \int_{\bar{x}_{i}^{N}}^{\bar{x}_{i+1}^{N}}\left(\bar{V}_{i}^{N}-v^{0}(x)\right) \rho^{0}(x) \varphi(x) d x
\end{aligned}
$$

Since $\bar{V}_{i}^{N}$ are bounded by $\left\|v^{0}\right\|_{\infty}$, we get similarly as for the convergence of $\hat{\rho}^{N}$ the estimate

$$
\begin{aligned}
& \quad \mid \sum_{i=0}^{N-1} \bar{V}_{i}^{N} \int_{\bar{x}_{i}^{N}}^{\bar{x}_{i+1}^{N}} \rho^{0}(x)\left(\varphi\left(\bar{x}_{i}^{N}\right)-\varphi(x)\right) d x \\
& \quad+\sum_{i=0}^{N-1} \bar{V}_{i}^{N} \frac{l_{N}}{\bar{x}_{i+1}^{N}-\bar{x}_{i}^{N}} \int_{\bar{x}_{i}^{N}}^{\bar{x}_{i+1}^{N}}\left(\varphi(x)-\varphi\left(\bar{x}_{i}^{N}\right)\right) d x \mid \\
& \leq l_{N} \frac{3}{2}\left\|\varphi^{\prime}\right\|_{\infty}\left\|v^{0}\right\|_{\infty}\left\|\rho^{0}\right\|_{\infty}\left(x_{\max }-x_{\min }\right) \underset{N \rightarrow+\infty}{\rightarrow} 0 .
\end{aligned}
$$

Now for the last term of the inequality, we have

$$
\left|\sum_{i=0}^{N-1} \int_{\bar{x}_{i}^{N}}^{\bar{x}_{i+1}^{N}}\left(\bar{V}_{i}^{N}-v^{0}(x)\right) \rho^{0}(x) \varphi(x) d x\right|
$$




$$
\begin{aligned}
& \leq \sum_{i=0}^{N-1} \int_{\bar{x}_{i}^{N}}^{\bar{x}_{i+1}^{N}}\left|\bar{V}_{i}^{N}-v^{0}(x)\right| \rho^{0}(x)|\varphi(x)| d x \\
& \leq \sum_{i=0}^{N-1} \int_{\bar{x}_{i}^{N}}^{\bar{x}_{i+1}^{N} \mid} \sup _{\left[\bar{x}_{i}^{N}, \bar{x}_{i+1}^{N}\right]} v^{0}-\inf _{\left[\bar{x}_{i}^{N}, \bar{x}_{i+1}^{N}\right]} v^{0}\left|\rho^{0}(x)\right| \varphi(x) \mid d x \\
& \leq\|\varphi\|_{\infty} \sum_{i=0}^{N-1}\left|\sup _{\left[\bar{x}_{i}^{N}, \bar{x}_{i+1}^{N}\right]} v^{0}-\inf _{\left[\bar{x}_{i}^{N}, \bar{x}_{i+1}^{N}\right]} v^{0}\right| \int_{\bar{x}_{i}^{N}}^{\bar{x}_{i+1}^{N}} \rho^{0}(x) d x \\
& \leq l_{N}\|\varphi\|_{\infty} T V\left(v^{0}\right) \underset{N \rightarrow+\infty}{\rightarrow} 0 .
\end{aligned}
$$

Thus we get

$$
\int_{\mathbb{R}} \hat{\rho}^{N}(0, x) \hat{v}^{N}(0, x) \varphi(x) d x \underset{N \rightarrow+\infty}{\rightarrow} \int_{\mathbb{R}} \rho^{0}(x) v^{0}(x) \varphi(x) d x .
$$

Similarly, we have

$$
\int_{\mathbb{R}} \hat{\rho}^{N}(0, x) \hat{p}^{N}(0, x) \varphi(x) d x \underset{N \rightarrow+\infty}{\rightarrow} \int_{\mathbb{R}} \rho^{0}(x) p^{0}(x) \varphi(x) d x .
$$

\section{3. $L^{\infty}$ and $B V$ estimates}

The dynamics of $x_{i}^{N}(t), V_{i}^{N}(t)$ and $p_{i}^{N}(t)$ described by (1.10), (1.11), implies the following properties.

Lemma 3.1. Let $\rho^{0}, v^{0}$ and $p^{0}$ satisfy (H1)-(H2). Then the functions $V_{i}^{N}(t)$ and $p_{i}^{N}(t)$ defined by (1.10), (1.11) satisfy

$$
\left|V_{i}^{N}(t)\right| \leq\left\|v^{0}\right\|_{\infty}, \quad\left|p_{i}^{N}(t)\right| \leq\left\|v^{0}\right\|_{\infty}+\left\|p^{0}\right\|_{\infty} .
$$

Moreover, it holds

$$
\begin{gathered}
T V\left(V^{N}(t, .)\right) \leq T V\left(V^{0}\right), \\
T V\left(p^{N}(t, .)\right) \leq T V\left(V^{0}\right)+T V\left(p^{0}\right) .
\end{gathered}
$$

Proof. The $L^{\infty}$ estimates (3.1) are deduced from the maximum principles

$$
\begin{gathered}
0 \leq V_{i}^{N}(t) \leq \max _{j} V_{j}^{N}(0) \leq \sup v^{0}, \\
0 \leq p_{i}^{N}(t) \leq \max _{j}\left(V_{j}^{N}(0)+p_{j}^{N}(0)\right) \leq \sup \left(v^{0}+p^{0}\right),
\end{gathered}
$$

which directly follow from the system dynamics.

The estimate (3.2) derives from the fact that between two interaction times $t_{m}$, the functions $t \mapsto V_{i}^{N}(t)$ are constant. At time $t_{m}$, for a collision between the $i_{m}$-th and the $\left(i_{m}+1\right)$-th particles, from (1.11) we have

$$
T V\left(V^{N}\left(t_{m}+, .\right)\right)=\sum_{i=0}^{N-1}\left|V_{i+1}^{N}\left(t_{m}+\right)-V_{i}^{N}\left(t_{m}+\right)\right|
$$




$$
\begin{aligned}
= & \sum_{i=0}^{i_{m}-1}\left|V_{i+1}^{N}\left(t_{m}+\right)-V_{i}^{N}\left(t_{m}+\right)\right|+\left|V_{i_{m}+1}^{N}\left(t_{m}+\right)-V_{i_{m}}\left(t_{m}+\right)\right| \\
& +\sum_{i=i_{m}+1}^{N-1}\left|V_{i+1}^{N}\left(t_{m}+\right)-V_{i}^{N}\left(t_{m}+\right)\right| \\
= & \sum_{i=0}^{i_{m}-1}\left|V_{i+1}^{N}\left(t_{m}-\right)-V_{i}^{N}\left(t_{m}-\right)\right|+\sum_{i=i_{m}+1}^{N-1}\left|V_{i+1}^{N}\left(t_{m}-\right)-V_{i}^{N}\left(t_{m}-\right)\right| \\
\leq & T V\left(V^{N}\left(t_{m}-, .\right)\right),
\end{aligned}
$$

thus proving (3.2). Notice that the variation which is lost for $V^{N}$ is transferred to $p^{N}$, thus giving $(3.3)$.

These properties clearly have the following consequences on the functions $\hat{\rho}^{N}, \hat{v}^{N}$ and $\hat{p}^{N}$ :

Proposition 3.2. We have the following estimates:

1. The functions $\hat{\rho}^{N}, \hat{v}^{N}$ and $\hat{p}^{N}$ are bounded in $L^{\infty}(] 0,+\infty[\times \mathbb{R})$.

2. Furthermore

$$
T V\left(\hat{v}^{N}(t, .)\right) \leq T V\left(v^{0}\right), \quad T V\left(\hat{p}^{N}(t, .)\right) \leq T V\left(v^{0}\right)+T V\left(p^{0}\right), \quad \forall N \in \mathbb{N} .
$$

Finally, notice that for all $x \in \mathbb{R}$ we have

$$
\left(\hat{\rho}^{N}(t, x)-\rho^{*}\right) \hat{p}^{N}(t, x)=0 .
$$

Indeed, this is true at $t=0$. Moreover

1. if $\hat{p}^{N}(0, x) \neq 0$ for $x \in\left[\bar{x}_{i}^{N}, \bar{x}_{i+1}^{N}\left[\right.\right.$, then $\hat{\rho}^{N}(t, x)=\rho^{*}$ for $x \in\left[x_{i}^{N}(t), x_{i+1}^{N}(t)[\right.$ for $t>0$;

2. when $\hat{p}^{N}$ passes from 0 to non-zero, as described by (1.11), then it is when $\hat{\rho}^{N}=\rho^{*}$ is satisfied.

\section{Convergence proofs}

\subsection{Study of the approximated equations}

We first start by studying the limit of the approximated equations.

Proposition 4.1. Let $\rho^{0}, v^{0}$ and $p^{0}$ satisfy (H1)-(H2). Then, for any $\varphi \in$ $C_{c}^{\infty}([0,+\infty[\times \mathbb{R})$, it holds

$$
-<\partial_{t} \hat{\rho}^{N}+\partial_{x}\left(\hat{\rho}^{N} \hat{v}^{N}\right), \varphi>\underset{N \rightarrow+\infty}{\rightarrow} \int_{\mathbb{R}} \rho^{0}(x) \varphi(0, x) d x
$$

and

$$
\begin{aligned}
-<\partial_{t} \hat{\rho}^{N}\left(\hat{v}^{N}+\hat{p}^{N}\right) & +\partial_{x}\left(\hat{\rho}^{N} \hat{v}^{N}\left(\hat{v}^{N}+\hat{p}^{N}\right)\right), \varphi> \\
& \underset{N \rightarrow+\infty}{\rightarrow} \int_{\mathbb{R}} \rho^{0}(x)\left(v^{0}+p^{0}\right)(x) \varphi(0, x) d x .
\end{aligned}
$$


Proof. Let $\varphi \in C_{c}^{1}([0,+\infty[\times \mathbb{R})$. We have

$$
\begin{aligned}
-<\partial_{t} \hat{\rho}^{N} & +\partial_{x}\left(\hat{\rho}^{N} \hat{v}^{N}\right), \varphi> \\
& =\int_{0}^{+\infty} \int_{\mathbb{R}} \hat{\rho}^{N}(t, x) \partial_{t} \varphi(t, x)+\hat{\rho}^{N}(t, x) \hat{v}^{N}(t, x) \partial_{x} \varphi(t, x) d x d t \\
& =\sum_{i=0}^{N-1} \int_{0}^{+\infty} y_{i}^{N}(t) \int_{x_{i}^{N}(t)}^{x_{i+1}^{N}(t)}\left(\partial_{t} \varphi(t, x)+V_{i}^{N}(t) \partial_{x} \varphi(t, x)\right) d x d t .
\end{aligned}
$$

Notice that

$$
\begin{aligned}
\frac{d}{d t} & \int_{x_{i}^{N}(t)}^{x_{i+1}^{N}(t)} \varphi(t, x) d x \\
& =\int_{x_{i}^{N}(t)}^{x_{i+1}^{N}(t)} \partial_{t} \varphi(t, x) d x+\dot{x}_{i+1}^{N}(t) \varphi\left(t, x_{i+1}^{N}(t)\right)-\dot{x}_{i}^{N}(t) \varphi\left(t, x_{i}^{N}(t)\right) \\
& =\int_{x_{i}^{N}(t)}^{x_{i+1}^{N}(t)} \partial_{t} \varphi(t, x) d x+V_{i+1}^{N}(t) \varphi\left(t, x_{i+1}^{N}(t)\right)-V_{i}^{N}(t) \varphi\left(t, x_{i}^{N}(t)\right),
\end{aligned}
$$

therefore

$$
\begin{aligned}
& -<\partial_{t} \hat{\rho}^{N}+\partial_{x}\left(\hat{\rho}^{N} \hat{v}^{N}\right), \varphi> \\
& =\sum_{i=0}^{N-1} \int_{0}^{+\infty} y_{i}^{N}(t)\left(\frac{d}{d t} \int_{x_{i}^{N}(t)}^{x_{i+1}^{N}(t)} \varphi(t, x) d x-V_{i+1}^{N}(t) \varphi\left(t, x_{i+1}^{N}(t)\right)\right. \\
& \left.\quad+V_{i}^{N}(t) \varphi\left(t, x_{i}^{N}(t)\right)+V_{i}^{N}(t)\left(\varphi\left(t, x_{i+1}^{N}(t)\right)-\varphi\left(t, x_{i}^{N}(t)\right)\right)\right) d t \\
& =\sum_{i=0}^{N-1} \int_{0}^{+\infty} y_{i}^{N}(t)\left(\frac{d}{d t} \int_{x_{i}^{N}(t)}^{x_{i+1}^{N}(t)} \varphi(t, x) d x-\left(V_{i+1}^{N}(t)-V_{i}^{N}(t)\right) \varphi\left(t, x_{i+1}^{N}(t)\right)\right) d t .
\end{aligned}
$$

Now

$$
\begin{aligned}
& \int_{0}^{+\infty} y_{i}^{N}(t) \frac{d}{d t} \int_{x_{i}^{N}(t)}^{x_{i+1}^{N}(t)} \varphi(t, x) d x d t \\
& \quad=-y_{i}^{N}(0) \int_{x_{i}^{N}(0)}^{x_{i+1}^{N}(0)} \varphi(0, x) d x-\int_{0}^{+\infty} \dot{y}_{i}^{N}(t) \int_{x_{i}^{N}(t)}^{x_{i+1}^{N}(t)} \varphi(t, x) d x d t
\end{aligned}
$$

which, with (1.13), gives

$$
\begin{aligned}
\int_{0}^{+\infty} & y_{i}^{N}(t) \frac{d}{d t} \int_{x_{i}^{N}(t)}^{x_{i+1}^{N}(t)} \varphi(t, x) d x d t \\
= & -y_{i}^{N}(0) \int_{x_{i}^{N}(0)}^{x_{i+1}^{N}(0)} \varphi(0, x) d x \\
& +\int_{0}^{+\infty} \frac{\left(y_{i}^{N}(t)\right)^{2}}{l_{N}}\left(V_{i+1}^{N}(t)-V_{i}^{N}(t)\right) \int_{x_{i}^{N}(t)}^{x_{i+1}^{N}(t)} \varphi(t, x) d x d t
\end{aligned}
$$


Furthermore

$$
\begin{aligned}
\varphi\left(t, x_{i+1}^{N}(t)\right) & =\frac{1}{x_{i+1}^{N}(t)-x_{i}^{N}(t)} \int_{x_{i}^{N}(t)}^{x_{i+1}^{N}(t)} \varphi\left(t, x_{i+1}^{N}(t)\right) d x \\
& =\frac{y_{i}^{N}(t)}{l_{N}} \varphi\left(t, x_{i+1}^{N}(t)\right) d x .
\end{aligned}
$$

Reporting (4.4) and (4.5) in (4.3), we obtain

$$
\begin{aligned}
- & <\partial_{t} \hat{\rho}^{N}+\partial_{x}\left(\hat{\rho}^{N} \hat{v}^{N}\right), \varphi> \\
& =\int_{0}^{T_{\varphi}} \Delta_{N}(t) d t-\sum_{i=0}^{N-1} y_{i}^{N}(0) \int_{x_{i}^{N}(0)}^{x_{i+1}^{N}(0)} \varphi(0, x) d x \\
& =\int_{0}^{T_{\varphi}} \Delta_{N}(t) d t-\int_{0}^{+\infty} \int_{\mathbb{R}} \hat{\rho}^{N}(0, x) \varphi(0, x) d x d t
\end{aligned}
$$

where

$$
\Delta_{N}(t)=\sum_{i=0}^{N-1} \frac{\left(y_{i}^{N}(t)\right)^{2}}{l_{N}}\left(V_{i+1}^{N}(t)-V_{i}^{N}(t)\right) \int_{x_{i}^{N}(t)}^{x_{i+1}^{N}(t)}\left(\varphi(t, x)-\varphi\left(t, x_{i+1}^{N}(t)\right)\right) d x
$$

with $T_{\varphi}$ such that $\varphi(t, x)=0$ for $t \geq T_{\varphi}$. Now we have

$\left|\int_{x_{i}^{N}(t)}^{x_{i+1}^{N}(t)}\left(\varphi(t, x)-\varphi\left(t, x_{i+1}^{N}(t)\right)\right) d x\right| \leq \frac{\left\|\varphi^{\prime}\right\|_{\infty}}{2}\left(x_{i}^{N}(t)-x_{i+1}^{N}(t)\right)^{2}=\frac{\left\|\varphi^{\prime}\right\|_{\infty} l_{N}^{2}}{2\left(y_{i}^{N}(t)\right)^{2}}$, thus

$$
\left.\left|\Delta_{N}(t)\right| \leq \frac{\left\|\varphi^{\prime}\right\|_{\infty} l_{N}}{2} \sum_{i=0}^{N-1} \mid V_{i+1}^{N}(t)-V_{i}^{N}(t)\right) \mid \leq \frac{\left\|\varphi^{\prime}\right\|_{\infty} l_{N}}{2} T V\left(v^{0}\right),
$$

and

$$
\left|\int_{0}^{T_{\varphi}} \Delta_{N}(t) d t\right| \leq \frac{\left\|\varphi^{\prime}\right\|_{\infty} l_{N}}{2} T_{\varphi} T V\left(v^{0}\right) \underset{N \rightarrow+\infty}{\rightarrow} 0 .
$$

Finally, we use Proposition 2.1 to conclude to (4.1).

For the second equation, we have

$$
\begin{aligned}
& -<\partial_{t}\left(\hat{\rho}^{N}\left(\hat{v}^{N}+\hat{p}^{N}\right)\right)+\partial_{x}\left(\hat{\rho}^{N} \hat{v}^{N}\left(\hat{v}^{N}+\hat{p}^{N}\right)\right), \varphi> \\
& =\int_{0}^{+\infty} \int_{\mathbb{R}} \hat{\rho}^{N}(t, x)\left(\hat{v}^{N}+\hat{p}^{N}\right)(t, x)\left(\partial_{t} \varphi(t, x)+\hat{v}^{N}(t, x) \partial_{x} \varphi(t, x)\right) d x d t \\
& =\sum_{i=0}^{N-1} \int_{0}^{+\infty} y_{i}^{N}(t)\left(V_{i}^{N}(t)+p_{i}^{N}(t)\right) \int_{x_{i}^{N}(t)}^{x_{i+1}^{N}(t)}\left(\partial_{t} \varphi(t, x)+V_{i}^{N}(t) \partial_{x} \varphi(t, x)\right) d x d t .
\end{aligned}
$$

Notice that $V_{i}^{N}(t)+p_{i}^{N}(t)$ is constant with respect to $t$. Indeed, when there is no collision $\dot{V}_{i}^{N}(t)=0$ and $\dot{p}_{i}^{N}(t)=0$ and at a collision time $t_{m}$,

$$
\begin{aligned}
V_{i_{m}}^{N}\left(t_{m}+\right)+p_{i_{m}}^{N}\left(t_{m}^{+}\right) & =V_{i_{m}+1}^{N}\left(t_{m}-\right),+V_{i_{m}}^{N}\left(t_{m}-\right)-V_{i_{m}+1}^{N}\left(t_{m}-\right)+p_{i_{m}}^{N}\left(t_{m}-\right) \\
& =V_{i_{m}}^{N}\left(t_{m}-\right)+p_{i_{m}}^{N}\left(t_{m}-\right) .
\end{aligned}
$$

Thus we get the convergence (4.2) as for the first equation. 


\subsection{Compactness estimates for $\hat{\rho}^{N}$}

To go further, a key point is to obtain some compactness for $\hat{\rho}^{N}$.

Proposition 4.2. Let $\rho^{0}$ and $v^{0}$ satisfy (H1)-(H2). For any $\phi \in C_{c}^{\infty}(\mathbb{R})$, there exists $C_{\phi}>0$ such that for any $N \in \mathbb{N}$ and any $s, t \in[0, T]$, it holds

$$
\left|\int_{\mathbb{R}}\left(\hat{\rho}^{N}(t, x)-\hat{\rho}^{N}(s, x)\right) \phi(x) d x\right| \leq C_{\phi}|t-s| .
$$

Therefore, up to a subsequence, there exists $\rho \in L^{\infty}(] 0, T[\times \mathbb{R})$ such that $\hat{\rho}^{N} \rightarrow \rho$ in $C\left([0, T], L_{w *}^{\infty}\left(\mathbb{R}_{x}\right)\right)$, i.e.

$$
\forall \Gamma \in L^{1}(\mathbb{R}), \quad \sup _{t \in[0, T]}\left|\int_{\mathbb{R}}\left(\hat{\rho}^{N}-\rho\right)(t, x) \Gamma(x) d x\right| \underset{k \rightarrow+\infty}{\rightarrow} 0 .
$$

Proof. In the formulation (4.6), we take $\varphi(t, x)=\Gamma_{R}(t) \phi(x)$ with $\Gamma_{R}$ with a compact support in $] 0,+\infty\left[\right.$ and we make $\Gamma_{R} \rightarrow \mathbf{I}_{[s, t]}$ when $R \rightarrow+\infty$, it gives

$$
\int_{\mathbb{R}}\left(\hat{\rho}^{N}(t, x)-\hat{\rho}^{N}(s, x)\right) \phi(x) d x+\int_{s}^{t} \int_{\mathbb{R}} \hat{\rho}^{N} \hat{u}^{N} \partial_{x} \phi=\int_{s}^{t} \tilde{\Delta}_{N}(\sigma) d \sigma
$$

for $\varphi$ where

$$
\tilde{\Delta}_{N}(t)=\sum_{i=0}^{N-1} \frac{\left(y_{i}^{N}(t)\right)^{2}}{l_{N}}\left(V_{i+1}^{N}(t)-V_{i}^{N}(t)\right) \int_{x_{i}^{N}(t)}^{x_{i+1}^{N}(t)}\left(\phi(x)-\phi\left(x_{i+1}^{N}(t)\right)\right) d x
$$

Similarly as in Section 4.1, we have

$$
\left|\int_{s}^{t} \tilde{\Delta}_{N}(\sigma) d \sigma\right| \leq|t-s| \frac{\left\|\phi^{\prime}\right\|_{\infty} l_{N}}{2} T V\left(v^{0}\right) .
$$

Furthermore, from Proposition 3.2,

$$
\left|\int_{s}^{t} \int_{\mathbb{R}} \hat{\rho}^{N} \hat{v}^{N} \partial_{x} \phi d x d \sigma\right| \leq|t-s| \rho^{*}\left\|v^{0}\right\|_{\infty} \int_{\mathbb{R}}|\phi| d x
$$

then

$$
\begin{aligned}
& \left|\int_{\mathbb{R}}\left(\hat{\rho}^{N}(t, x)-\hat{\rho}^{N}(s, x)\right) \phi(x) d x\right| \\
& \leq|t-s|\left(\frac{\left\|\phi^{\prime}\right\|_{\infty}\left\|\rho^{0}\right\|_{1}}{2 N} T V\left(v^{0}\right)+\rho^{*}\left\|v^{0}\right\|_{\infty} \int_{\mathbb{R}}|\phi| d x\right) .
\end{aligned}
$$

To conclude, we use the following Lemma 4.3 proved in [4].

Lemma 4.3. Let $\left(n_{k}\right)_{k \in \mathbb{N}}$ be a bounded sequence in $L^{\infty}(] 0, T[\times \mathbb{R})$ which satisfies: for all $\phi \in C_{c}^{\infty}(\mathbb{R})$, the sequence $\left(\int_{\mathbb{R}} n_{k}(t, x) \phi(x) d x\right)_{k}$ is uniformly Lipschitz continuous on $[0, T]$, i.e. $\exists C_{\phi}>0$,

$$
\forall k \in \mathbb{N}, \quad \forall s, t \in[0, T], \quad\left|\int_{\mathbb{R}}\left(n_{k}(t, x)-n_{k}(s, x)\right) \phi(x) d x\right| \leq C_{\phi}|t-s| .
$$


Then, up to a subsequence, there exists $n \in L^{\infty}(] 0, T[\times \mathbb{R})$ such that $n_{k} \rightarrow n$ in $C\left([0, T], L_{w *}^{\infty}\left(\mathbb{R}_{x}\right)\right)$, i.e.

$$
\forall \Gamma \in L^{1}(\mathbb{R}), \quad \sup _{t \in[0, T]}\left|\int_{\mathbb{R}}\left(n_{k}-n\right)(t, x) \Gamma(x) d x\right| \underset{k \rightarrow+\infty}{\rightarrow} 0 .
$$

We have a similar result from the second equation, that is to say:

Proposition 4.4. Let $\rho^{0}, v^{0}$ and $p^{0}$ satisfy (H1)-(H2). For any $\phi \in C_{c}^{\infty}(\mathbb{R})$, there exists $C_{\phi}>0$ such that for any $N \in \mathbb{N}$ and any $s, t \in[0, T]$, we have

$$
\left|\int_{\mathbb{R}}\left(\left(\hat{\rho}^{N}\left(\hat{v}^{N}+\hat{p}^{N}\right)\right)(t, x)-\left(\hat{\rho}^{N}\left(\hat{v}^{N}+\hat{p}^{N}\right)\right)(s, x)\right) \phi(x) d x\right| \leq C_{\phi}|t-s| .
$$

Then, up to a subsequence, there exists $q \in L^{\infty}(] 0, T[\times \mathbb{R})$ such that $\hat{\rho}^{N}\left(\hat{u}^{N}+\right.$ $\left.\hat{p}^{N}\right) \rightarrow q$ in $C\left([0, T], L_{w *}^{\infty}\left(\mathbb{R}_{x}\right)\right)$, i.e.

$$
\forall \Gamma \in L^{1}(\mathbb{R}), \quad \sup _{t \in[0, T]}\left|\int_{\mathbb{R}}\left(\hat{\rho}^{N}\left(\hat{v}^{N}+\hat{p}^{N}\right)-q\right)(t, x) \Gamma(x) d x\right| \underset{k \rightarrow+\infty}{\rightarrow} 0 .
$$

Proof. This time, we have, for any $\phi \in C_{c}^{\infty}(\mathbb{R})$,

$$
\begin{gathered}
\int_{\mathbb{R}}\left(\left(\hat{\rho}^{N}\left(\hat{v}^{N}+\hat{p}^{N}\right)\right)(t, x)-\left(\hat{\rho}^{N}\left(\hat{v}^{N}+\hat{p}^{N}\right)\right)(s, x)\right) \phi(x) d x \\
=-\int_{s}^{t} \int_{\mathbb{R}} \hat{\rho}^{N} \hat{v}^{N}\left(\hat{v}^{N}+\hat{p}^{N}\right) \partial_{x} \phi+\int_{s}^{t} \bar{\Delta}_{N}(\sigma) d \sigma
\end{gathered}
$$

where

$\bar{\Delta}_{N}(t)=\sum_{i=0}^{N-1}\left(V_{i}^{N}(t)+p_{i}^{N}(t)\right) \frac{\left(y_{i}^{N}(t)\right)^{2}}{l_{N}}\left(V_{i+1}^{N}(t)-V_{i}^{N}(t)\right) \int_{x_{i}^{N}(t)}^{x_{i+1}^{N}(t)}\left(\phi(x)-\phi\left(x_{i+1}^{N}(t)\right)\right) d x$.

We have now

$$
\left|\int_{s}^{t} \bar{\Delta}_{N}(\sigma) d \sigma\right| \leq|t-s|\left\|\phi^{\prime}\right\|_{\infty} l_{N} T V\left(v^{0}\right)\left(\left\|v^{0}\right\|_{\infty}+\left\|p^{0}\right\|_{\infty}\right)
$$

using furthermore (3.1). Then we get

$$
\begin{aligned}
& \left.\mid \int_{\mathbb{R}}\left(\left(\hat{\rho}^{N}\left(\hat{v}^{N}+\hat{p}^{N}\right)\right)(t, x)-\left(\hat{\rho}^{N}\left(\hat{v}^{N}+\hat{p}^{N}\right)\right)(s, x)\right)\right) \phi(x) d x \mid \\
& \leq|t-s|\left(\left\|\phi^{\prime}\right\|_{\infty} \frac{\left\|\rho^{0}\right\|_{1}}{N} T V\left(v^{0}\right)+2 \rho^{*}\left\|v^{0}\right\|_{\infty} \int_{\mathbb{R}}|\phi| d x\right)\left(\left\|v^{0}\right\|_{\infty}+\left\|p^{0}\right\|_{\infty}\right) .
\end{aligned}
$$

We conclude using the previous Lemma 4.3.

\subsection{Convergence to the limit equations}

We need now to pass to the limit in the product terms. We recall the following result, which is the key point of the proof to pass to the limit in the products.

Lemma 4.5. Let us assume that $\left(n_{k}\right)_{k \in \mathbb{N}}$ is a bounded sequence in $L^{\infty}(] 0, T[\times \mathbb{R})$ that tends to $n$ in $L_{w *}^{\infty}(] 0, T[\times \mathbb{R})$, and satisfies for any $\phi \in C_{c}^{\infty}\left(\mathbb{R}_{x}\right)$,

$$
\int_{\mathbb{R}}\left(n_{k}-n\right)(t, x) \phi(x) d x \underset{k \rightarrow+\infty}{\rightarrow} 0,
$$


either $i)$ a.e. $t \in] 0, T\left[\right.$ or ii) in $L^{1}(] 0, T[t)$.

Let us also assume that $\left(\omega_{k}\right)_{k \in \mathbb{N}}$ is a bounded sequence in $L^{\infty}(] 0, T[\times \mathbb{R})$ that tends to $\omega$ in $L_{w *}^{\infty}(] 0, T[\times \mathbb{R})$, and such that for all compact interval $K=[a, b]$, there exists $C>0$ such that the total variation (in $x$ ) of $\omega_{k}$ over $K$ satisfies

$$
\forall k \in \mathbb{N}, \quad T V_{K}\left(\omega_{k}(t, .)\right) \leq C .
$$

Then, $n_{k} \omega_{k} \rightarrow n \omega$ in $L_{w *}^{\infty}(] 0, T[\times \mathbb{R})$ as $k \rightarrow+\infty$.

Remark 4.6. This is a result of compensated compactness, which uses the compactness in $x$ for $\left(\omega_{k}\right)_{k}$ given by (4.11) and the weak compactness in $t$ for $\left(n_{k}\right)_{k}$ given by (4.10) to pass to the weak limit in the product $n_{k} \omega_{k}$. We can refer to [3] for a complete proof, even in the case where

$$
\forall k \in \mathbb{N}, \quad T V_{K}\left(\omega_{k}(t, .)\right) \leq C\left(1+\frac{1}{t}\right),
$$

which is more general. Notice that the total variation bound (in $\mathrm{x}$ ) of $\omega$ over $K$ is also satisfied thanks to the lower semi-continuity to the BV norm.

We are now able to obtain the limit result.

Proof of Theorem 1.2. Since $\left(\hat{\rho}^{N}\right)_{N},\left(\hat{v}^{N}\right)_{N},\left(\hat{p}^{N}\right)_{N}$ are bounded in $L^{\infty}$, there exists $(\rho, u, p)$ such that

$$
\hat{\rho}^{N} \rightarrow \rho, \quad \hat{v}^{N} \rightarrow v, \quad \hat{p}^{N} \rightarrow p \quad \text { in } L_{w *}^{\infty}(] 0,+\infty[\times \mathbb{R}) .
$$

By Proposition 4.2, we also have $\hat{\rho}^{N} \rightarrow \rho$ in $C\left([0, T], L_{w *}^{\infty}\left(\mathbb{R}_{x}\right)\right)$.

Using Proposition 3.2, we get that the sequences $\left(\hat{v}^{N}(t, .)\right)_{N}$ and $\left(\hat{p}^{N}(t, .)\right)_{N}$ are uniformly bounded in BV with respect to t.

We can then apply the Lemma 4.5, which gives that $\hat{\rho}^{N} \hat{v}^{N} \rightarrow \rho v$ in $L_{w *}^{\infty}(] 0, T[\times \mathbb{R})$ and $\hat{\rho}^{N} \hat{p}^{N} \rightarrow \rho p$ in $L_{w *}^{\infty}(] 0, T[\times \mathbb{R})$. Therefore the (4.1) of Proposition 4.1 gives that

$$
-<\partial_{t} \rho+\partial_{x}(\rho v), \varphi>=\int_{\mathbb{R}} \rho^{0}(x) \varphi(0, x) d x .
$$

By Proposition 4.4, there exists $q \in L^{\infty}(10, T[\times \mathbb{R})$ such that, up to a subsequence, $\hat{\rho}^{N}\left(\hat{v}^{N}+\hat{p}^{N}\right) \rightarrow q$ in $C\left([0, T], L_{w *}^{\infty}\left(\mathbb{R}_{x}\right)\right)$. By uniqueness of the limit $q=\rho(v+p)$. We apply now Lemma 4.5, which gives that $\hat{\rho}^{N} \hat{v}^{N}\left(\hat{v}^{N}+\right.$ $\left.\hat{p}^{N}\right) \rightarrow \rho v(v+p)$ in $L_{w *}^{\infty}(] 0, T[\times \mathbb{R})$. Therefore the (4.2) of Proposition 4.1 gives that

$$
-<\partial_{t} \rho(v+p)+\partial_{x}(\rho v(v+p)), \varphi>=\int_{\mathbb{R}} \rho^{0}(x)\left(v^{0}(x)+p^{0}(x)\right) \varphi(0, x) d x .
$$

Now we pass to the limit in $0 \leq \hat{\rho}^{N} \leq \rho^{*}, \hat{p}^{N} \geq 0,\left(\hat{\rho}^{N}-\rho^{*}\right) \hat{p}^{N}=0$ to get the constraints and conclude the proof.

\section{Acknowledgment}

This work was achieved while the first author was in secondment at Inria Sophia Antipolis. 


\section{References}

[1] A. Aw, A. Klar, T. Materne, and M. Rascle. Derivation of continuum traffic flow models from microscopic follow-the-leader models. SIAM J. Appl. Math., 63(1):259-278, 2002.

[2] A. Aw and M. Rascle. Resurrection of "second order" models of traffic flow. SIAM J. Appl. Math., 60:916-938, 2000.

[3] F. Berthelin. Existence and weak stability for a pressureless model with unilateral constraint. Math. Models Methods Appl. Sci., 12(2):249-272, 2002.

[4] F. Berthelin and D. Broizat. A model for the evolution of traffic jams in multilane. Kinet. Relat. Models, 5(4):697-728, 2012.

[5] F. Berthelin, P. Degond, M. Delitala, and M. Rascle. A model for the formation and evolution of traffic jams. Arch. Ration. Mech. Anal., 187(2):185-220, 2008.

[6] R. M. Colombo, F. Marcellini, and M. Rascle. A 2-phase traffic model based on a speed bound. SIAM J. Appl. Math., 70(7):2652-2666, 2010.

[7] R. M. Colombo and E. Rossi. On the micro-macro limit in traffic flow. Rend. Semin. Mat. Univ. Padova, 131:217-235, 2014.

[8] M. Di Francesco and M. D. Rosini. Rigorous derivation of nonlinear scalar conservation laws from follow-the-leader type models via many particle limit. Arch. Ration. Mech. Anal., 217(3):831-871, 2015.

[9] M. D. Francesco, S. Fagioli, and M. D. Rosini. Many particle approximation of the Aw-Rascle-Zhang second order model for vehicular traffic. Math. Biosci. Eng., 14(1):127-141, 2017.

[10] M. D. Francesco, S. Fagioli, M. D. Rosini, and G. Russo. Deterministic particle approximation of the Hughes model in one space dimension. Kinet. Relat. Models, 10(1):215-237, 2017.

[11] P. Goatin and F. Rossi. A traffic flow model with non-smooth metric interaction: well-posedness and micro-macro limit. To appear on Comm. Math. Sci., 2017.

[12] H. M. Zhang. A non-equilibrium traffic model devoid of gas-like behavior. Transp. Research Part B: Method., 36(3):275-290, 2002.

Florent Berthelin

Laboratoire J. A. Dieudonné, UMR 7351 CNRS,

Université Côte d'Azur, LJAD, CNRS, Inria,

Université de Nice Sophia-Antipolis, Parc Valrose,

06108 Nice cedex 2, France

e-mail: Florent.Berthelin@unice.fr

Paola Goatin

Inria Sophia Antipolis - Méditerranée, Université Côte d'Azur, Inria, CNRS, LJAD, 2004 route des Lucioles - BP 93, 06902 Sophia Antipolis Cedex, France e-mail: paola.goatin@inria.fr 